\title{
In vivo observations provide insight into roles of eosinophils and epithelial cells in asthma
}

\author{
Carl Persson \\ Affiliation: Laboratory Medicine University Hospital, Lund, Sweden. \\ Correspondence: Carl Persson, Laboratory Medicine University Hospital, Lund, Sweden. \\ E-mail: carl.perssonamed.lu.se
}

@ERSpublications

Exploratory in vivo research approaches produce unexpected discoveries and novel understanding independent of currently accepted paradigms http://bit.ly/2YgLdnF

Cite this article as: Persson C. In vivo observations provide insight into roles of eosinophils and epithelial cells in asthma. Eur Respir J 2019; 54: 1900470 [https://doi.org/10.1183/13993003.00470-2019].

\begin{abstract}
Observations in vivo in patients, supported by guinea-pig in vivo data, take centre stage in this perspective. Its objective is to highlight dichotomies between asthma features observed in vivo and accepted views involving cell/molecular biology research paradigms. For example, increased bronchial epithelial permeability is now considered a major paradigm and trait of asthma, yet, absorption of inhaled tracers has not been increased in vivo in asthma. Such maintained barrier function in exudative asthma reflects in vivo asymmetry of the epithelial lining as barrier between outside and inside world of molecules and cells. In desquamatory asthma, maintained epithelial tightness may be explained by in vivo demonstrations of exceedingly patchy epithelial loss, prompt creation of plasma-derived provisional barriers, and high-speed epithelial regeneration. Acknowledged protein/peptide secretion by epithelial cells in vitro is contrasted here with a dominant, unidirectional movement in vivo of plasma-derived proteins/ peptides (including antimicrobial peptides) to the surface of an intact epithelial lining. Furthermore, longstanding claims that epithelium-produced adenosine is a mediator of asthma are eroded by observations in vivo in asthmatics. Notions concerning activation/fate of mucosal tissue eosinophils illustrate additional distinctions between accepted views and in vivo patient observations. Finally, in vitrobased paradigms preaching defect epithelial regeneration and increased permeability in pathogenesis of asthma are contrasted with experimental in vivo observations of exaggerated epithelial regeneration, which is multipathogenic in its own right. In conclusion, unexpected and challenging in vivo observations in recent decades underpin novel insights into mucosal mechanisms in asthma.
\end{abstract}




\section{Introduction}

For $\sim 150$ years, authors have championed the importance of the bronchial epithelium in asthma. Epithelial shedding and associated exudative events were among the first focal areas [1]. The potential for interactions between eosinophils and asthmatic epithelium was noted soon after the specific staining of these cells had emerged. In addition, 100 years ago it was speculated that epithelial-derived factors could be important in asthma [1]. The early intriguing reports dealt largely with observations of sputum and histopathology.

Whereas the epithelium and the eosinophil remain at the forefront in asthma research, revelations of late are overwhelmingly based on findings involving cultured cells and molecular biology approaches in vitro and in vivo. In addition, intriguing biological data have constituted a basis for build-up and support of currently accepted concepts on airway functions in health and asthma. We are taught about mechanisms of airway barriers, mucosal defence, eosinophil-epithelial inflammation, epithelial regeneration, and, by inference, pathogenesis of asthma. However, it appears that several paradigms have been firmly established without due consideration of observational in vivo data. This development may only in part reflect lack of well-validated attempts at translating in vitro data to the in vivo picture, but it does concern occasions when in vivo observations have not agreed with acknowledged views.

Towards the end of the 1990s it was widely declared that independent observation-based clinical research had nothing more to offer the medical investigator. The important revelations in that regard had already been made. However, not everyone agreed. In Lund, we were making several unexpected in vivo observations regarding airway microvascular, epithelial and eosinophil aspects $[2,3]$, so we had to disagree $[1,4,5]$. Consistent with their unexpected nature, most observations did not support the paradigms then in vogue. Thus, deeply rooted views and research paradigms were challenged by the novel ideas that we discussed. Our approach involved physiologically well-controlled in vivo conditions. However, probably contributing to a slow acceptance, we did not provide the usual supporting data from cell and mouse model molecular biology.

In this perspective the focus is foremost on observations in patients (denoted as human in vivo data), but with support from guinea-pig in vivo data (specified as such). Although short-term experiments in rodents may not completely reflect events in human chronic asthma, the guinea-pig models have advantages over mouse models with regard to the specific issues discussed in this review. Thus, human airway-like plasma exudation, eosinophil cytolysis and epithelial cell loss have clearly occurred in guinea-pig models, but not in mouse models $[6,7]$. In several other respects mouse models may provide attractive experimental opportunities [8].

Importantly, our continued analyses, based in no small part on an expanding source of patient in vivo data publicised by many different authors, have underpinned our ideas. Recent years have also seen a growing acceptance of the original in vivo findings and associated concepts. This is reflected by recent dissemination of reviews focusing on airway plasma exudation in health and disease [9] and on elimination [10, 11] and activation of eosinophils [12, 13]. Hence, it seemed timely to discuss the commonality of dichotomies between in vivo observations and accepted views. Several examples in the field of airway and asthma research are collated here with the objective to highlight controversies between in vivo observations and widely acknowledged paradigms. An additional aim is to promote exploratory in vivo research approaches. To these ends this perspective article discusses epithelial and eosinophilic aspects of the airway mucosa in vivo in asthma. Underscoring the present theme, this perspective further includes select aspects of medical history and pharmacology of anti-asthma drugs.

\section{Widened aspects of airway epithelium and eosinophils in asthma}

The physical barrier function of the airway epithelial lining is more complex than commonly discussed. For example, there is an asymmetry of the epithelial lining that needs emphasising. It stands out by its readiness to let through macromolecules in the outward direction without altering its tightness as an absorption barrier. The mechanisms involved in this unidirectional perviousness, including supporting human patient in vivo data, have been reviewed recently [9]. In the present perspective article, particularly intriguing examples of patient observations are added to reinforce the apparently controversial view that an exudative disease such as asthma can have a tight barrier with a normal rate of absorption of inhaled molecules (figure 1).

Early in vitro studies on transepithelial migration of granulocytes reported a marked asymmetry favouring the outward basolateral-to-apical direction; furthermore, the transepithelial passage left unharmed tight junctions $[14,15]$. The studies may not have focused on elimination of granulocytes. However, such in vitro observations agree with demonstrations of a role in vivo of transepithelial migration (figure 1 ) as a swift and silent way of ridding the airway mucosa of granulocytes in asthma [10, 11].

Less translational, but widely acknowledged in vitro observations have claimed that death of eosinophils through apoptosis, followed by phagocytosis, is the true fate of eosinophils at inflammation resolution [16]. 


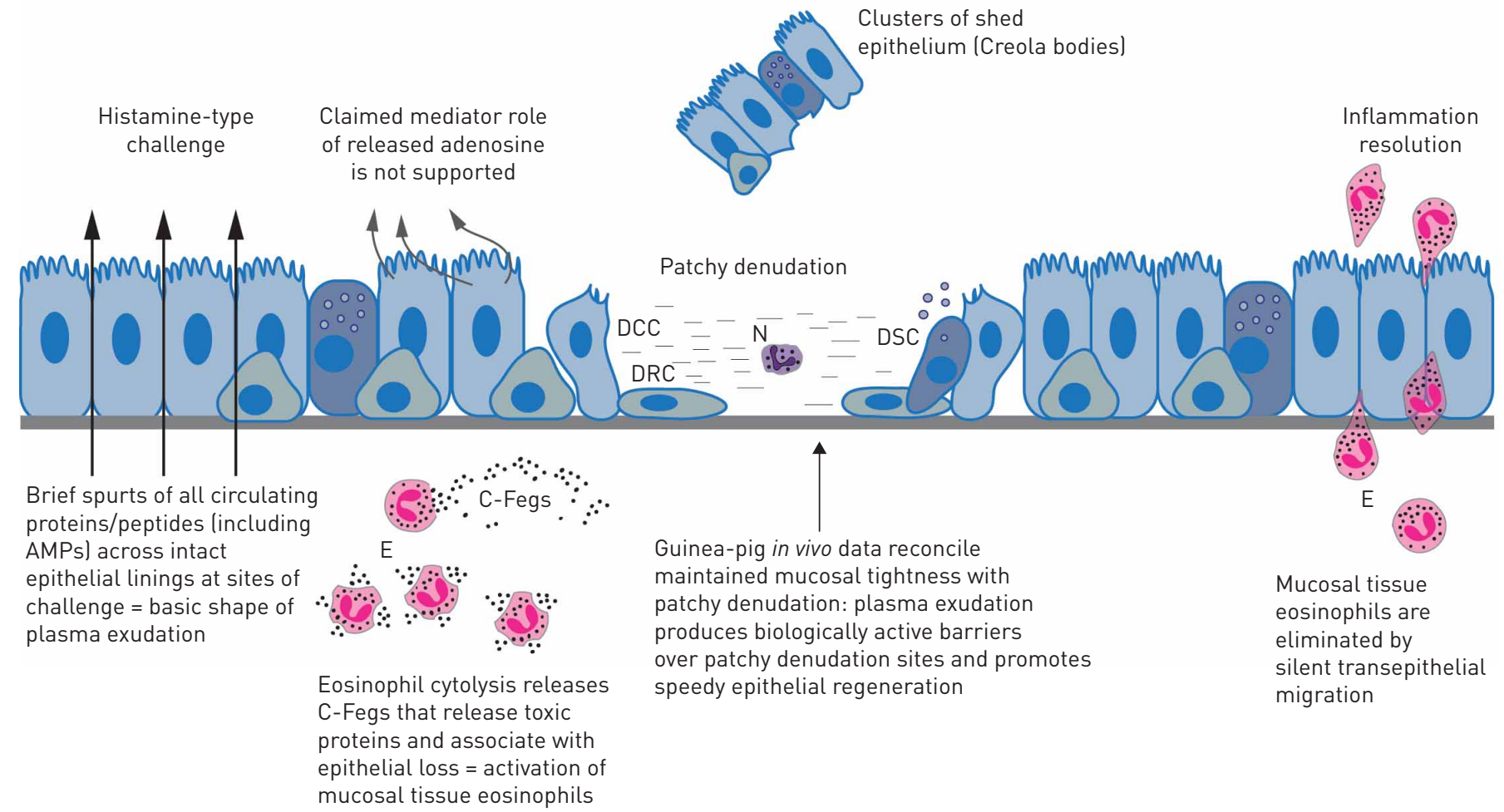

FIGURE 1 Epithelial and eosinophil features demonstrated by and consistent with in vivo observations in asthma patients. Features of the epithelial lining, such as lack of increased absorption, exudative response to histamine-type challenge, patchiness of epithelial loss, events following patchy desquamation and involvement in inflammation resolution are indicated above the epithelium. Features involving the superficial microcirculation and mucosal eosinophils are indicated below the epithelium. The illustrated epithelial regeneration events at denudation patches represent observations that so far have been made in guinea-pig airways in vivo. Other features in this figure represent in vivo observations made in human asthma as well as in guinea-pigs. As discussed in this article, there are distinct dichotomies between roles of the depicted in vivo events and established paradigms that are largely built on observations in vitro. DCC: dedifferentiating ciliated cell; DRC: dedifferentiated regeneration cells; DSC: dedifferentiating secretory cell; N: neutrophils; E: eosinophils; AMPs: antimicrobial peptides; C-Fegs: clusters of free eosinophil granules.

Yet, this rapidly accepted and long-standing concept has not been confirmed in vivo. Indeed, the proven in vivo mode of death of airway mucosal tissue eosinophils in asthma takes another shape and is a striking mode of activation of these cells in vivo $[12,13]$.

In recent years the role of epithelial lining cells as a biologically active barrier has received much attention [17]. It is clear that epithelial cells have capacity to release many molecules of interest in airway defence and disease. Some of the epithelial-derived molecules are promoted as mediators of asthma and are now targets for anti-asthma drugs. As discussed here, in vivo efficacy of drugs is pivotal in deciding which mediator is important.

This review further underscores the possibility that plasma-derived rather than epithelial-derived molecules may be responsible for biological activities of the airway mucosal surface in vivo. The examples concern plasma exudation-induced appearance of major defence and repair molecules including antimicrobial peptides on the surface of an intact bronchial epithelial lining in vivo in health and asthma (figure 1). A pronounced role also emerges at sites of epithelial denudation (largely guinea-pig in vivo data; figure 1) where exuded plasma produces a provisional barrier, which at the same time creates a milieu that promotes speedy epithelial regeneration.

\section{Absorption of inhaled molecules is not increased in asthma patients, not even in asthma caused by epithelium-toxic chemicals; are these data enough to erode the current idea of increased epithelial permeability as a paradigm of pathogenesis of asthma?}

Aspects of a maintained epithelial barrier in asthma, not included in a previous review [9], are discussed here. Toluene diisocyanate (TDI) is an epithelium-toxic chemical [18] identified as a major culprit in occupational asthma. Expectedly, inhalation of TDI produces acute effects including plasma exudation [19]. 
However, relatively recent studies $[20,21]$ demonstrate lack of increased absorption permeability in painters exposed to isocyanates, with or without occupational asthma. Painters exposed to TDI rather exhibited a tendency at reduced absorption permeability [21]. These findings significantly expand a series of in vivo data published in the 1990s indicating that asthmatic individuals in general do not exhibit increased permeability to inhaled molecules (summarised in [9]). Indeed, absorption permeability has actually been reduced [9] in studies (in asthma and in seasonal allergic rhinitis) where concomitant mucociliary clearance of tracers has been controlled. In 2018, absorption of inhaled mannitol was examined in human asthma and found to be no different from healthy subjects [22]. Thus, in vivo observations involving many cohorts and different permeability tracers have disproven the idea of increased inward permeability as a basic defect in asthma (figure 1). Notwithstanding these patient data, increased epithelial permeability to inhaled agents remains a widely acknowledged research paradigm and pathogenic trait of asthma [23, 24].

\section{Inflammatory stimulus-induced acute airways plasma exudation: to what extent is} this associated with increased absorption-permeability of the epithelial lining?

At baseline there is a minor degree of bronchial mucosal leakage of plasma proteins. This leakage differs from the acute plasma exudation response [9] by being subject to sieving [25]. Thus, under stable conditions small proteins may preferentially appear in airway surface liquids. This consideration makes large plasma proteins, notably $\alpha_{2}$-macroglobulin, a better index of acute plasma exudation than albumin [9]. Furthermore, especially at baseline leakage, charged entities of mucosal extracellular matrix such as glycosaminoglycans will likely bind plasma proteins [26] and thus reduce their availability for epithelial passage. Reversely, plasma proteins may bind cell-produced proteins in asthma. Thus, inducement of acute exudation of plasma has brought tissue-dwelling eosinophil cationic proteins to the mucosal surface [9].

Several aspects of acute inflammatory challenge-induced plasma exudation warrant attention: the plasticity of the airway epithelial lining combined with a hydraulic mechanism evidently lets through plasma macromolecules irrespective of size and charge in one direction without altering its function as a barrier against movements of molecules in the opposite direction [9] (figure 1). Furthermore, the acute plasma exudation response is repeatable and reproducible. In addition, it is short-lasting unless the challenge is aggravated [9].

Collateral evidence supports a swift and barrier-maintaining mode of bringing all plasma molecules to the intact mucosal surface: airways plasma exudation responses have not caused mucosal oedema or increased lymph transport of plasma tracers in guinea-pigs. Hence, epithelial transmission of plasma at acute responses has not created a need for drainage of the mucosal tissue of extravasated proteins [9]. The experimental in vivo data on plasma exudation are further compatible with asthma being a disease that exhibits plasma exudation $[9,27]$ without increased absorption of inhaled molecules, and where evidence of actual mucosal oedema is scarce [9]. Inferentially, the ubiquitous idea that airways plasma exudation always reflects epithelial derangement and a generally increased perviousness of the mucosal barrier is not supported.

\section{Antimicrobial peptides and major defence and repair proteins on the surface of an intact bronchial mucosa in vivo: are they components of plasma exudation or produced by local airway cells?}

Reflecting the non-sieved nature of the acute plasma exudation response, major antimicrobial players, including high and low molecular weight kininogens of the contact system [28] are exuded as components of plasma exudation in human airways [29]. Similarly, as recently reviewed in some detail [9], complement proteins, fibrinogen, fibronectin, etc. appear on the mucosal surface as components of a plasma exudate. To summarise, the basic shape of acute plasma exudation has the properties of a powerful defence mechanism of the intact mucosal surface. Thus, roles of plasma exudation deserve consideration in current discussions of first-line mucosal defence.

In an elegant study involving atopic asthma patients, Liu et al. [30] demonstrated that cathelicidin, a major antimicrobial peptide (AMP) appeared on the bronchial surface at allergen exposure exclusively as a result of plasma exudation. Considering the ample opportunities for plasma exudation to occur at any site along the human airways it thus appears that plasma exudation could be the important mechanism that brings cathelicidin, and possibly other AMPs as well, to the surface of an intact mucosa (figure 1). This possibility now emerges as a novel aspect in the cell culture-dominated field of AMP research.

In a study of sputum levels of several antimicrobial peptides/proteins in chronic obstructive pulmonary disease (COPD) it was only cathelicidin that was increased in association with colonisation of pathogenic bacteria at exacerbations [31]. Plasma exudation occurs in both asthma and COPD, especially at exacerbations [32]. Hence, it seems likely, as in the study by Liu et al. [30], that cathelicidin was a component of exuded plasma in the COPD study [31]. 
Resolution of airway eosinophilic inflammation: elimination of eosinophils through apoptosis/phagocytosis that occurs in vitro, or epithelial transmission that occurs in vivo?

A widely acknowledged concept states that elimination of airway mucosal leukocytes occurs through apoptosis followed by phagocytosis [16]. Thus, apoptosis of eosinophils is hailed as a major mechanism of inflammation resolution in asthma. Further supporting this idea, it is believed that corticosteroids and select biological drugs operate by inducing eosinophil apoptosis [16]. However, apoptotic eosinophils remain to be demonstrated in diseased airway tissues in vivo whether steroid treatment is given or not $[13,33,34]$. Thus, the popular idea that corticosteroids operate by inducing eosinophil apoptosis is not borne out by in vivo observations. Similarly, no compelling in vivo data are known to support the common view that novel biologics, antagonising interleukin-5, induce eosinophil apoptosis in vivo in asthma $[33,35,36]$. There is now minimal attention to lack of in vivo support for a role of apoptosis in the pharmacology of these major anti-asthma drug classes.

Based on sputum cell analysis, eosinophil apoptosis was reported in 1996 to occur in human asthma [37]. This interesting report needs confirmation. However, a major question concerns the meaning of any leukocyte apoptosis in airway lumen material? By necessity, every claimed apoptotic eosinophil in the airway lumen must have been alive as long as it dwelled in the diseased airway tissue, or it could not have migrated into the lumen. Notably, in this discussion of modes of elimination of eosinophils the lack of apoptotic eosinophils concerns the living blood-perfused airway tissue.

During the decades when the apoptosis hypothesis became established, patient in vivo data indicated that two other fates of bronchial tissue eosinophils were important [11, 13], yet they were little noticed: the silent fate of epithelial transmission and its pathogenic counterpart fate, primary cytolysis of eosinophils (cytolysis is discussed later). Large numbers of tissue eosinophils can be eliminated by transepithelial migration without distorting the airway epithelial barrier $[34,38]$ (figure 1). This mode agrees with published, but rarely discussed observations at resolution of inflammation in vivo in asthma [10, 11]. Interpretation of granulocyte numbers in the air space would depend on the phase of disease processes, whether inflammation is developing, persistent or resolving.

The epithelial transmission of eosinophils can occur randomly between epithelial cells [34, 38]. This aspect would be important both for inflammation resolution and for the ability of these cells to be active in first line antimicrobial defence at any site of mucosal surface attack. The defence aspect has been particularly well demonstrated with regard to neutrophils emerging on the epithelial surface of urinary bladders [39]. In the gut mucosa the interest in epithelial crossing of neutrophils has an additional focus on anchorage of the leukocytes to the apical epithelial membrane in defence and inflammation [40]. HULBERT et al. [41], who underscored roles in first line mucosal defence, claimed that neutrophils were transmitted across the airway epithelial lining in a train fashion at certain sites. Studies seem warranted to better define roles in vivo of epithelial transmission of granulocytes both for defence operations at the surface of an intact airway mucosa and for silent elimination of these cells at inflammation resolution.

\section{Cytolysis of eosinophils and occurrence of clusters of free eosinophil granules: is this an artefact or a major mode of eosinophil activation in vivo?}

Occurrence and potential importance of eosinophil cytolysis tally with the present theme of original in vivo discoveries. Whereas apoptotic eosinophils could qualify for a phantom phenomenon, another shape of eosinophil death, primary cytolysis, abounds in vivo in diseased tissues [13, 42, 43]. Many tissue eosinophils in asthma are thus eliminated by primary cytolysis (without involvement of apoptosis). Importantly, this mode of death entails ultimate activation and potential pathogenic effects of these cells in diseased airways, including an intriguing association with epithelial shedding [13, 43] (figure 1).

Prior to and in parallel with the decades of hegemony of the apoptosis mechanism, the cytolysis mode of eosinophil death was considered an artefact or, perhaps most commonly, not considered at all [44]. A turning point conquering the disbelief was the understanding and the demonstration beyond doubt that the cytolysis was an inducible, non-artefact in vivo phenomenon [44]. As such it was readily identified in vivo by occurrence of clusters of free eosinophil granules (C-Fegs) in the airway mucosa [13, 45] (figure 1). The ultrastructural features of cytolysis include chromatolysis and cell membrane rupture, allowing the spilling of cell nucleus and cytoplasm contents. Most conspicuous are the C-Fegs, containing and releasing potent proteins [44].

C-Fegs are intriguingly associated with childhood and adult asthma including apparent associations with epithelial sloughing and severity of the disease [13, 43]. A role of eosinophils in epithelial derangement and shedding has further been suggested based on epithelium-toxic actions of granule proteins [46, 47] known to be released from C-Fegs in vivo in human diseased airways [13]. Bandeira-Melo and Weller [48] have 
significantly expanded the research area by demonstrating that C-Fegs can be separately regulated as release-competent organelles.

Occurrence and potential importance of primary cytolysis of eosinophils are increasingly being accepted $[12,13,43]$. One piece in this development is the recent report by leading champions of the eosinophil apoptosis paradigm acknowledging that apoptotic eosinophils may rarely occur in human diseased airway tissue $[49,50]$. There have also been publications reporting that molecular mechanisms of eosinophil apoptosis may actually have dealt with primary cytolysis of eosinophils [33].

The current adoption of the paradigm of eosinophil cytolysis by experts on the biology of eosinophil apoptosis [51] is both telling and welcome. A controversy may remain regarding the role of primary cytolysis in formation of eosinophil extracellular nets $[49,52]$. However, the current degree of acceptance of eosinophil cytolysis as a major mechanism should advance our knowledge of the molecular biology of eosinophil cytolysis. Perhaps it is time to complement the mere counting of blood eosinophils [53] with a test of their propensity to undergo primary cytolysis. The aim would be to move towards precision in identifying patients whose disease is eosinophil-driven.

\section{Highly active plasma-derived barriers over a denuded, but intact, basement membrane cater for safe and speedy epithelial regeneration in vivo: might exaggerated epithelial regeneration, rather than the current paradigm of defect repair, characterise asthma?}

LEMARCHAND et al. [54] reported that acute asthma differed from stable asthma by exhibiting a transient increase in bronchial clearance of inhaled tracer molecules. Hence, in the acute condition, where epithelial shedding may be much increased [55], bronchial permeability to inhaled molecules may be increased. This possibility needs confirmation. However, the transient nature of the permeability increase, together with the lack of increased absorption in stable asthma (discussed earlier) would argue against the currently established notion of defect epithelial repair in asthma [56, 57]. Accordingly, it was recently suggested that asthma may be a disease where exaggerated epithelial regeneration activities operate to oppose escalating epithelial barrier breaks once epithelial denudation has occurred [9].

Asthma-like denudation in vivo in guinea-pigs is non-sanguineous and involves no injury to the basement membrane [58]. At such denudation sites epithelial regeneration starts promptly [58]. Studies of epithelial shedding-repair in vivo, where the basement membrane has been injured, have recorded a considerably delayed start of regeneration and a relatively slow re-establishment of a new cell cover $[59,60]$. This is the case even if commendable attempts have been made to produce tiny areas of denudation [60].

Lack of bleeding does not mean lack of wound protection. Guinea-pig in vivo studies demonstrate that promptly following non-sanguineous removal of epithelium from the intact basement membrane, plasma proteins exude and produce a highly active barrier kept together at the denudation site by a plasma-derived fibrin-fibronectin net. This provisional barrier is continuously supplied by bulk plasma and leukocytes, including activated neutrophils $[58,61]$. A milieu of exuded plasma proteins may be a prerequisite for speedy repair. Furthermore, a provisional, plasma-derived barrier at patchy sites of shedding, together with high-speed epithelial restitution, probably contributes to a maintained absorption barrier in asthma (figure 1).

Following asthma-like denudation in guinea-pig airways in vivo, all types of epithelial cells bordering the spot of exposed basement membrane, notably including columnar ciliated and secretory cells, promptly dedifferentiated into poorly differentiated, flattened, mesenchymal- and basal cell-like cells [58]. By a joint effort of all neighbouring cells, the restitution of a cell cover would thus be optimised in vivo (figure 1). This observation was novel at the time. The regeneration cells moved in a tethered fashion and at a high speed in a plasma exudate milieu [58]. By $15 \mathrm{~min}$ the front of migrating regeneration cells had moved $30-50 \mu \mathrm{m}$. Basal cells have commonly been considered stem cells responsible for epithelial regeneration in the lung [62]. By advanced techniques it has now been confirmed that local epithelial cell types other than basal cells have capacities to participate in airway epithelial regeneration [63]. Apparently, this recent development has not needed the support provided by the original in vivo observations.

\section{Micrographs illustrating extensive denudation may reflect areas with numerous tiny denudation spots; is patchy epithelial sloughing key to maintained mucosal tightness in vivo in asthma?}

Increased sloughing of epithelium in asthma is evident from analyses of sputum samples. Epithelial derangement and denudation have also been illustrated in micrographs of childhood and adult asthma [64, 65]. A current discussion concerns the possibility that denudation graphs may represent epithelial fragility just as much as actual denudation [66]. Indeed, it may well be both. Experimental airway challenges in guinea-pigs and dogs with allergen and dry air have produced many tiny sites of denudation $[42,67,68]$, most clearly 
demonstrated in helicopter views of tissue whole-mounts [42]. Sectioning of airways with many small spots of denudation has produced much increased areas of complete denudation [42], suggesting the novel possibility that common denudation micrographs of asthmatic bronchi could represent areas scattered with patchy denudation spots.

Patchiness of epithelial loss in asthma is an infrequently discussed phenomenon. However, its occurrence is supported by in vivo observations of epithelial cells clumped together in sputum. This feature was repeatedly observed at the turn of the 19th century [1]. It was studied in greater detail in the 1960s. Honouring the name of one of his patients, NAYLOR [55] named clumps of epithelial cells occurring in sputum from asthma patients Creola bodies (figure 1). Creola bodies are commonly defined by their content of the easily recognisable ciliated cells. However, other types of epithelial cells may also be present. Although selective loss of ciliated cells may occur in asthma [69], small clusters of both columnar and basal epithelial cells may leave the basement membrane together [46] to produce Creola bodies.

\section{Pathogenesis of asthma: exaggerated epithelial regeneration rather than increased permeability?}

Epithelial shedding seems particularly pronounced at exacerbations [55, 70]. Thus, NAYLOR [55] observed Creola bodies in nearly all asthmatics that delivered sputum during asthma exacerbations or the week after an asthma attack. The number of Creola bodies in only two smears could be well over 100 [55]. Hence, numerous sites of shedding were probably involved, suggesting that in acute and severe asthma there is an exceedingly patchy mode of epithelial denudation (figure 1). Supporting the association between epithelial loss and disease severity, patients with increased numbers of Creola bodies tended to have a longer stay in hospital [55]. In agreement, OGata et al. [70] demonstrated that a sputum Creola body score correlated with both sputum levels of eosinophil cationic proteins and the number of hospital days needed for remission after asthma attacks.

YAMADA and colleagues [71, 72] have made interesting observations on occurrence of Creola bodies in sputum samples obtained from infants. One study focused on 23 hospitalised, wheezing infants with a mean age of $\sim 5$ months. They were divided into two groups depending on presence or absence of Creola bodies. In the first month following discharge asthma symptoms were much higher in the group positive for Creola bodies. $12(80 \%)$ of these children, but none in the group that lacked Creola bodies, were diagnosed with infantile asthma during a 2-year follow-up period [71].

In a second study, YAmada and YoshiHara [72] examined occurrence of Creola bodies in infants admitted with acute respiratory syncytial virus (RSV) infection. Potential roles of infection with RSV in inception of asthma have received much attention, but data in this field have not been consistent [73]. In their study of RSV-infected infants, YAmada and Yoshinara [72] demonstrated that only those who presented with Creola bodies in aspirated sputum exhibited a significant risk of developing recurrent wheezing and asthma during follow-up at 2 and 5 years (relative risk of 3 and 7, respectively). Hence, the authors strengthened their idea of a prognostic value of epithelial shedding in predicting future development of asthma.

Lethal RSV infections of infants have been characterised by pronounced shedding of epithelial cells. Sloughed epithelium together with admixed fibrin and some mucus have occluded the bronchial lumen in these cases [74]. Of note, the epithelial sloughing in cross-sections was patchy [74]. This finding further tallies with demonstrations of quite patchy occurrence of infections in bronchial epithelial cells exposed to rhinovirus [75].

As reviewed elsewhere, the mere removal of epithelial cells from a small area of intact basement membrane in vivo in guinea-pig airways is followed by several events of interest in relation to features of asthma [9, 76, 77]. Reflecting common regeneration stages epithelial cell remodelling occurs at regeneration sites [58]. Importantly, additional effects of inflammatory as well as remodelling nature are induced: 1) plasma is exuded;2) mucus is secreted; 3) neutrophils are recruited and activated; 4) eosinophils already present in local airway tissues undergo primary cytolysis; 5) proliferation of subepithelial fibrocytes/smooth muscle is induced; and 6) by repeated epithelial denudation-regeneration sequences the reticular basement membrane is thickened $[43,76$, 78]. Molecular pathways involved in the regeneration-induced effects listed earlier have not been determined. Whereas such a limitation may prevent establishment of a strong research paradigm, it does not invalidate the in vivo based idea of costly epithelial regeneration in asthma as a pathogenic factor in its own right.

\section{Anti-asthma drugs and proposed mediators of asthma emanating from stressed epithelium: is the claimed role of adenosine eroded by in vivo observations in asthma?}

Since their arrival, the modus operandi of anti-asthma drug classes has naturally been explained in the light of contemporary ideas of disease features. When asthma was considered a disease of reduced nerve 
activity, strong coffee (containing methylxanthines) was active because it was a stimulant [79]. When asthma was thought to reflect "vasomotor ataxia", adrenalin acted because it was a vasoconstrictor [1], etc. Now drug and disease mechanisms are being linked based on cell and molecular biology observations. However, the discussion on roles of eosinophil apoptosis suggests that not all currently accepted views on pharmacological mechanisms might stand the test of critical in vivo evaluations.

Several autacoids released from stressed and injured airway epithelium have attracted pharmacological interest. Thymic stromal lymphopoietin (TSLP) is known to be induced by human epithelial cells at rhinoviral infection $[80,81]$ and injury repair $[82,83]$. Since clinical efficacy of a biologic anti-TSLP drug in exacerbating asthma was demonstrated [84], TSLP now stands out as a drug target. Adenosine emanates from injured epithelium [85] and has for a long time been viewed as an important mediator of asthma [86]. The idea is claimed to be supported by cell and molecular biology data (of unknown clinical relevance) and, more concretely, by propositions that theophylline, a universal adenosine antagonist, is active in asthma through its antagonism of adenosine [86]. This dogma was not helped by the "surprise" arrival of enprofylline, a xanthine chemically very close to theophylline, but dramatically different regarding adenosine antagonism and extrapulmonary actions [87].

The distinct profile of enprofylline in humans in vivo reflected its lack of inhibition of adenosine-induced effects [87]. Regarding anti-asthma efficacy, ranging from inhibition of allergen challenge-induced late-phase reaction to improvement over placebo at maintenance treatment, the two xanthines were both clinically efficacious and to the same extent [88-90]. The only difference in asthma was detected when theophylline, but not enprofylline, preferentially inhibited adenosine challenge-induced bronchoconstriction in patients [91]. Unfortunately, in seminal discussions these data have been turned around and referred to as "preferential inhibitory effect of intravenous enprofylline on adenosine-induced bronchoconstriction" [86]. The crucial fact is the opposite.

Reflecting its efficacy combined with lack of adenosine antagonism, enprofylline reduced bronchoconstriction produced by histamine and adenosine challenges equally. However, theophylline, being a potent adenosine antagonist, was the drug that preferentially inhibited the adenosine-induced effect [91]. Hence, the two xanthines, at relevant plasma concentrations, were successfully used as tools to critically assess involvement of adenosine in asthma. As agreed in the original publication [91], the data together with the clinical efficacy of enprofylline [88-90] seriously eroded the idea that adenosine was an important mediator of asthma. In summary, clinical effects together with pharmacological features of anti-asthma drugs demonstrate that TSLP is an important epithelium-derived player in asthma, whereas a role of adenosine is not supported. Increased expression of TSLP in repairing epithelium [83] is further consistent with a pathogenic role of exaggerated epithelial regeneration in asthma.

\section{Select early contributions to our understanding of asthma and its treatment may not have received due attention; tribute to Salter and Solis-Cohen}

I think we can still learn from Henry Hyde Salter (1823-1871), an astute original observer of numerous important features of asthma. Without the need of molecular explanations his original descriptions of "asthma, its pathology and treatment" (cited in $[79,92]$ ) have lasting value. It is no coincidence that he could contribute so much. Expressing that colleagues "had done more in the way of reading each other's books than scrutinising their own patients", Salter strongly advocated "simple reading of nature". Thus, he was clear in his criticism of the common occurrence of "unquestioning inheritance and adoption of received notions". His teachings also involved encouraging advice: "respect for authority, however high, should never be pushed to concessions of anything positively observed". However, several of Salter's astute accounts may not have been widely noticed. This concerns his observations relating to major anti-asthma drug principles [79]. In addition, his detailed observations of asthma exacerbations evoked by common cold may have been missed and confused with his important notes on the influence of cold air [92]. For nearly a century Solomon Solis-Cohen, who gave an intriguing description of glucocorticosteroid-like therapeutic features of oral intake of "adrenal substance" in asthma, was incorrectly considered to have made a first demonstration of effects of adrenaline [1]. These examples of medical history aspects may be significant by revealing how easy it is to miss essential in vivo observations. As discussed above, there is a risk that also some of the currently held notions and associated research paradigms are similarly unsupported by well-established data in vivo.

\section{How can research paradigms thrive in the face of contradictory in vivo observations?}

Developing fast since the final decades of 1900s biotechnical advances at molecular and cellular levels have created an explosive development of biological research opportunities. Naturally, research approaches have been selected where the novel techniques could be well applied. Thus, innovative cell culture experiments 
often combined with versatile mouse models came to dominate the airway research scene, from which an avalanche of new data emerged. The possibility that actual disease mechanisms might not be reflected in the available test systems received less attention. Hence, a risk of imbalance in medical research became evident [5].

New biological information was not only intriguing; thanks to increasingly available molecular schemes, data could also be communicated with proposed chains of events whereby biology was transformed into apparent medical importance. Complementary demonstration of individual molecules in patient airways might then suffice to produce high-impact definitive reports. The language advantage inherent in the reductive approaches cannot be underestimated. Unsupportive observational in vivo data in patients may have been available. However, lacking in molecular explanations, they carried less weight. Linguistic disadvantage may in part explain why in vivo observations would not be considered convincing enough to create, or refute, paradigms.

The new biology (studies using cell and molecular biology techniques) also achieved hegemony in part based on overstatements inferring that what could be learnt by observation-based in vivo events had already been well established. Embarking on the increasingly available novel opportunities with purported medical importance became exceedingly rewarding. Under such circumstances unbiased and de novo considerations of what has been observed in vivo in patients might not receive the highest priority. It seemed acceptable to be history-less regarding disease-relevant observations in vivo.

Communication of biological data has involved explicit promises. Thus, suggested paths towards treatment opportunities have flourished in the literature. However, those who take proposed novel drug targets seriously would be wise to validate the reported data carefully. It was recently noted that $>60 \%$ of drug targets reported in high-impact journals were not even reproducible [93]. Such flaws may be telling about the attraction of discoveries at the molecular level for researchers and journals alike.

Importantly, the limitations of popular concepts such as those discussed in this article should not detract from the fact that molecular biology and cell studies have been and will be successful in creating novel medically important concepts, also leading to significant treatment opportunities. One such advance, perhaps even exceeding expectations of experts in the field [81], is the recent demonstration that a biological drug, specifically antagonising epithelial-derived TSLP, inhibits exacerbations of asthma [84].

\section{Conclusion}

This perspective article discusses a variety of epithelial and eosinophil features of the airways in asthma. The focus is on in vivo observations that underpin ideas that deviate from or complement current notions and research paradigms. Inferentially, this review highlights the potential importance of unexpected and challenging in vivo discoveries. The specific issues reflect a biased selection of discussion points. However, the fact that an outlook largely limited to personal encounters has identified these instances of dichotomies between in vivo observations and commonly accepted paradigms suggests that the phenomenon has a degree of general applicability.

Conflict of interest: C. Persson has nothing to disclose.

\section{References}

1 Persson CG. Centennial notions of asthma as an eosinophilic, desquamative, exudative, and steroid-sensitive disease. Lancet 1997; 350: 1021-1024.

2 Persson CG, Erjefält JS, Andersson M, et al. Epithelium, microcirculation, and eosinophils - new aspects of the allergic airway in vivo. Allergy 1997; 52: 241-255.

3 Persson CG. In vivo veritas: the continuing importance of discoveries in complex biosystems. Thorax 1996; 51: 441-443.

Persson C. Clinical research, or classical clinical research? Nat Med 1999; 5: 714-715.

Persson CG, Erjefalt JS, Uller L, et al. Unbalanced research. Trends Pharmacol Sci 2001; 22: 538-541. Persson CG, Erjefält JS, Korsgren M, et al. The mouse trap. Trends Pharmacol Sci 1997; 18: 465-467.

Persson CG. Con: mice are not a good model of human airway disease. Am J Respir Crit Care Med 2002; 166: 6-7.

8 Bonniaud P, Fabre A, Frossard N, et al. Optimising experimental research in respiratory diseases: an ERS statement. Eur Respir J 2018; 51: 1702133.

9 Persson C. Airways exudation of plasma macromolecules: innate defense, epithelial regeneration, and asthma. J Allergy Clin Immunol 2019; 143: 1271-1286.

10 Persson C, Uller L. Transepithelial exit of leucocytes: inflicting, reflecting or resolving airway inflammation? Thorax 2010; 65: 1111-1115.

11 Persson C, Uller L. Resolution of leucocyte-mediated mucosal diseases. A novel in vivo paradigm for drug development. Br J Pharmacol 2012; 165: 2100-2109.

12 Persson C, Uller L. Primary lysis of eosinophils as a major mode of activation of eosinophils in human diseased tissues. Nat Rev Immunol 2013; 13: 902. 
13 Persson C, Uller L. Theirs but to die and do: primary lysis of eosinophils and free eosinophil granules in asthma. Am J Respir Crit Care Med 2014; 189: 628-633.

14 Liu L, Mul FP, Lutter R, et al. Transmigration of human neutrophils across airway epithelial cell monolayers is preferentially in the physiologic basolateral-to-apical direction. Am J Respir Cell Mol Biol 1996; 15: 771-780.

15 Samejima Y, Masuyama K, Ishikawa T. Transepithelial migration of eosinophils in experimental nasal allergy in guinea pigs. Auris Nasus Larynx 1988; 15: 33-42.

16 Ilmarinen P, Moilanen E, Kankaanranta H. Regulation of spontaneous eosinophil apoptosis - a neglected area of importance. J Cell Death 2014; 7: 1-9.

17 López-Rodríguez JC, Benedé S, Barderas R, et al. Airway epithelium plays a leading role in the complex framework underlying respiratory allergy. J Investig Allergol Clin Immunol 2017; 27: 346-355.

18 Pham DL, Trinh TH, Ban GY, et al. Epithelial folliculin is involved in airway inflammation in workers exposed to toluene diisocyanate. Exp Mol Med 2017; 49: e395.

19 Vandenplas O, Delwiche JP, Staquet P, et al. Pulmonary effects of short-term exposure to low levels of toluene diisocyanate in asymptomatic subjects. Eur Respir J 1999; 13: 1144-1150.

20 Kaya M, Salan A, Tabakoğlu E, et al. The bronchoalveolar epithelial permeability in house painters as determined by Tc-99m DTPA aerosol scintigraphy. Ann Nucl Med 2003; 17: 305-308.

21 Cerci SS, Ozturk O, Sutcu R, et al. Evaluation of the effects of toluene inhalation on alveolar epithelial permeability by 99mTc-DTPA inhalation scintigraphy in automobile painters. Nucl Med Commun 2008; 29: 45-52.

22 Georas S, Ransom N, Hillman S, et al. The leaky lung test: a pilot study using inhaled mannitol to measure airway barrier function in asthma. J Asthma 2018: 10.1080/02770903.2018.1536145.

23 Georas SN, Rezaee F. Epithelial barrier function: at the front line of asthma immunology and allergic airway inflammation. J Allergy Clin Immunol 2014; 134: 509-520.

24 Ganesan S, Comstock AT, Sajjan US. Barrier function of airway tract epithelium. Tissue Barriers 2013; 1: e24997.

25 Schoonbrood DF, Lutter R, Habets FJ, et al. Analysis of plasma-protein leakage and local secretion in sputum from patients with asthma and chronic obstructive pulmonary disease. Am J Respir Crit Care Med 1994; 150: 1519-1527.

26 Gill S, Wight TN, Frevert CW. Proteoglycans: key regulators of pulmonary inflammation and the innate immune response to lung infection. Anat Rec 2010; 293: 968-981.

27 Desai D, Gupta S, Siddiqui S, et al. Sputum mediator profiling and relationship to airway wall geometry imaging in severe asthma. Respir Res 2013; 14: 17.

28 Frick IM, Björck L, Herwald H. The dual role of the contact system in bacterial infectious disease. Thromb Haemost 2007; 98: 497-502.

29 Baumgarten CR, Naclerio RM, Togias AG, et al. Kininogens in nasal secretions after allergen challenge. Adv Exp Med Biol 1986; 198: 189-192.

30 Liu MC, Xiao HQ, Brown AJ, et al. Association of vitamin D and antimicrobial peptide production during late-phase allergic responses in the lung. Clin Exp Allergy 2012; 42: 383-391.

31 Parameswaran GI, Sethi S, Murphy TF. Effects of bacterial infection on airway antimicrobial peptides and proteins in COPD. Chest 2011; 140: 611-617.

32 Persson C, Uller L. Roles of plasma exudation in asthma and COPD. Clin Exp Allergy 2009; 39: 1626-1629.

33 Persson C. Eosinophil apoptosis-inducing drugs risk worsening rather than resolving asthma. J Allergy Clin Immunol 2015; 135: 1662.

34 Uller L, Emanuelsson CA, Andersson M, et al. Early phase resolution of mucosal eosinophilic inflammation in allergic rhinitis. Respir Res 2010; 11: 54.

35 Rosenberg HF, Phipps S, Foster PS. Eosinophil trafficking in allergy and asthma. J Allergy Clin Immunol 2007; 119: $1303-1310$

36 Bochner BS, Kiwamoto T, Katoh T, et al. Reply: To PMID 25497369. J Allergy Clin Immunol 2015; 135: 1662-1663.

37 Woolley KL, Gibson PG, Carty K, et al. Eosinophil apoptosis and the resolution of airway inflammation in asthma. Am J Respir Crit Care Med 1996; 154: 237-243.

38 Erjefält JS, Uller L, Malm-Erjefält $\mathrm{M}$, et al. Rapid and efficient clearance of airway tissue granulocytes through transepithelial migration. Thorax 2004; 59: 136-143.

39 Hang L, Haraoka M, Agace WW, et al. Macrophage inflammatory protein-2 is required for neutrophil passage across the epithelial barrier of the infected urinary tract. I Immunol 1999; 162: 3037-3044.

40 Zhou G, Yu L, Fang L, et al. CD177 ${ }^{+}$neutrophils as functionally activated neutrophils negatively regulate IBD. Gut 2018; 67: 1052-1063.

41 Hulbert WC, Walker DC, Hogg JC. The site of leukocyte migration through the tracheal mucosa in the guinea pig. Am Rev Respir Dis 1981; 124: 310-316.

42 Erjefält JS, Korsgren M, Nilsson MC, et al. Prompt epithelial damage and restitution processes in allergen challenged guinea-pig trachea in vivo. Clin Exp Allergy 1997; 27: 1458-1470.

43 Persson C. Primary lysis of eosinophils in severe desquamative asthma. Clin Exp Allergy 2014; 44: 173-183.

44 Persson CG, Erjefält JS. "Ultimate activation" of eosinophils in vivo: lysis and release of clusters of free eosinophil granules (Cfegs). Thorax 1997; 52: 569-574.

45 Erjefält JS, Korsgren M, Nilsson MC, et al. Association between inflammation and epithelial damage-restitution processes in allergic airways in vivo. Clin Exp Allergy 1997; 27: 1344-1355.

46 Frigas E, Gleich GJ. The eosinophil and the pathophysiology of asthma. J Allergy Clin Immunol 1986; 77: 527-537.

47 Venge P. Eosinophils and respiratory disease. Respir Med 2001; 95: 168-169.

48 Bandeira-Melo C, Weller PF. Mechanisms of eosinophil cytokine release. Mem Inst Oswaldo Cruz 2005; 100: 73-81.

49 Persson C, Ueki S. Lytic eosinophils produce extracellular DNA traps as well as free eosinophil granules. J Allergy Clin Immunol 2018; 141: 1164.

50 Gevaert E, Yousefi S, Bachert C, et al. Reply. J Allergy Clin Immunol 2018; 141: 1164-1165.

51 Radonjic-Hoesli S, Wang X, de Graauw E, et al. Adhesion-induced eosinophil cytolysis requires the receptor-interacting protein kinase 3 (RIPK3)-mixed lineage kinase-like (MLKL) signaling pathway, which is counterregulated by autophagy. J Allergy Clin Immunol 2017; 140: 1632-1642. 
52 Ueki S, Konno Y, Takeda M, et al. Eosinophil extracellular trap cell death-derived DNA traps: their presence in secretions and functional attributes. J Allergy Clin Immunol 2016; 137: 258-267.

53 Kostikas K, Brindicci C, Patalano F. Blood eosinophils as biomarkers to drive treatment choices in asthma and COPD. Curr Drug Targets 2018; 19: 1882-1896.

54 Lemarchand P, Chinet T, Collignon MA, et al. Bronchial clearance of DTPA is increased in acute asthma but not in chronic asthma. Am Rev Respir Dis 1992; 145: 147-152.

55 Naylor B. The shedding of the mucosa of the bronchial tree in asthma. Thorax 1962; 17: 69-72.

56 Kicic A, Stevens PT, Sutanto EN, et al. Impaired airway epithelial cell responses from children with asthma to rhinoviral infection. Clin Exp Allergy 2016; 46: 1441-1455.

57 Sweerus K, Lachowicz-Scroggins M, Gordon E, et al. Claudin-18 deficiency is associated with airway epithelia barrier dysfunction and asthma. J Allergy Clin Immunol 2017; 139: 72-81.

58 Erjefält JS, Erjefält I, Sundler F, et al. In vivo restitution of airway epithelium. Cell Tissue Res 1995; 281: 305-316.

59 Keenan KP, Wilson TS, McDowell EM. Regeneration of hamster tracheal epithelium after mechanical injury. IV. Histochemical, immunocytochemical and ultrastructural studies. Virchows Arch B Cell Pathol Incl Mol Pathol 1983; 43: 213-240.

60 Kretschmer S, Pieper M, Klinger A, et al. Imaging of wound closure of small epithelial lesions in the mouse trachea. Am J Pathol 2017; 187: 2451-2460.

61 Erjefält JS, Erjefält I, Sundler F, et al. Microcirculation-derived factors in airway epithelial repair in vivo. Microvasc Res 1994; 48: 161-178.

62 Lynch TJ, Engelhardt JF. Progenitor cells in proximal airway epithelial development and regeneration. J Cell Biochem 2014; 115: 1637-1645.

63 Montoro DT, Haber AL, Biton M, et al. A revised airway epithelial hierarchy includes CFTR-expressing ionocytes. Nature 2018; 560: 319-324.

64 Laitinen LA, Heino M, Laitinen A, et al. Damage of the airway epithelium and bronchial reactivity in patients with asthma. Am Rev Respir Dis 1985; 131: 599-606.

65 Barbato A, Turato G, Baraldo S, et al. Epithelial damage and angiogenesis in the airways of children with asthma. Am J Respir Crit Care Med 2006; 174: 975-981.

66 Ordoñez C, Ferrando R, Hyde DM, et al. Epithelial desquamation in asthma: artifact or pathology? Am J Respir Crit Care Med 2000; 162: 2324-2329.

67 Davis MS, Freed AN. Repeated hyperventilation causes peripheral airways inflammation, hyperreactivity, and impaired bronchodilation in dogs. Am J Respir Crit Care Med 2001; 164: 785-789.

68 Davis MS, Schofield B, Freed AN. Repeated peripheral airway hyperpnea causes inflammation and remodeling in dogs. Med Sci Sports Exerc 2003; 35: 608-616.

69 Montefort S, Roberts JA, Beasley R, et al. The site of disruption of the bronchial epithelium in asthmatic and non-asthmatic subjects. Thorax 1992; 47: 499-503.

70 Ogata H, Motojima S, Fukuda T, et al. [Creola bodies and the eosinophil cationic protein in sputum in acute asthmatic attacks with respect to their clinical significance]. Arerugi 1990; 39: 1567-1575.

71 Yamada Y, Yoshihara S, Arisaka O. Creola bodies in wheezing infants predict the development of asthma. Pediatr Allergy Immunol 2004; 15: 159-162.

72 Yamada Y, Yoshihara S. Creola bodies in infancy with respiratory syncytial virus bronchiolitis predict the development of asthma. Allergol Int 2010; 59: 375-380.

73 Jartti T, Gern JE. Role of viral infections in the development and exacerbation of asthma in children. J Allergy Clin Immunol 2017; 140: 895-906.

74 Johnson JE, Gonzales RA, Olson SJ, et al. The histopathology of fatal untreated human respiratory syncytial virus infection. Mod Pathol 2007; 20: 108-119.

75 Mosser AG, Brockman-Schneider R, Amineva S, et al. Similar frequency of rhinovirus-infectible cells in upper and lower airway epithelium. J Infect Dis 2002; 185: 734-743.

76 Persson CG, Erjefält JS, Erjefält I, et al. Epithelial shedding - restitution as a causative process in airway inflammation. Clin Exp Allergy 1996; 26: 746-755.

77 Erjefält JS, Sundler F, Persson CG. Eosinophils, neutrophils, and venular gaps in the airway mucosa at epithelia removal-restitution. Am J Respir Crit Care Med 1996; 153: 1666-1674.

78 Erjefält JS, Persson CG. Airway epithelial repair: breathtakingly quick and multipotentially pathogenic. Thorax 1997; 52: 1010-1012.

79 Persson CG. On the medical history of xanthines and other remedies for asthma: a tribute to HH Salter. Thorax 1985; 40: 881-886

80 Calvén J, Yudina Y, Hallgren O, et al. Viral stimuli trigger exaggerated thymic stromal lymphopoietin expression by chronic obstructive pulmonary disease epithelium: role of endosomal TLR3 and cytosolic RIG-I-like helicases. Innate Immun 2012; 4: 86-99.

81 Uller L, Persson C. Viral induced overproduction of epithelial TSLP: role in exacerbations of asthma and COPD? J Allergy Clin Immunol 2018; 142: 712.

82 Allakhverdi Z, Comeau MR, Jessup HK, et al. Thymic stromal lymphopoietin is released by human epithelial cells in response to microbes, trauma, or inflammation and potently activates mast cells. J Exp Med 2007; 204: 253-258.

83 Uller L, Andersson M, Yudina Y, et al. Epithelial repair and expression of TSLP and EGFR in nasal polyp explants. Am J Respir Crit Care Med 2009; 179: A4952.

84 Corren J, Parnes JR, Wang L, et al. Tezepelumab in adults with uncontrolled asthma. N Engl J Med 2017; 377: 936-946.

85 Poernbacher I, Vincent JP. Epithelial cells release adenosine to promote local TNF production in response to polarity disruption. Nat Commun 2018; 9: 4675.

86 Holgate ST. The Quintiles Prize Lecture 2004. The identification of the adenosine A2B receptor as a novel therapeutic target in asthma. Br J Pharmacol 2005; 145: 1009-1015.

87 Persson CG. Development of safer xanthine drugs for treatment of obstructive airways disease. J Allergy Clin Immunol 1986; 78: 817-824.

88 Lunell E, Svedmyr N, Andersson KE, et al. A novel bronchodilator xanthine apparently without adenosine receptor antagonism and tremorogenic effect. Eur J Respir Dis 1983; 64: 333-339. 
89 Pauwels R, Van Renterghem D, Van der Straeten M, et al. The effect of theophylline and enprofylline on allergen-induced bronchoconstriction. J Allergy Clin Immunol 1985; 76: 583-590.

90 Chapman KR, Boucher S, Hyland RH, et al. A comparison of enprofylline and theophylline in the maintenance therapy of chronic reversible obstructive airway disease. J Allergy Clin Immunol 1990; 85: 514-521.

91 Clarke H, Cushley MJ, Persson CG, et al. The protective effects of intravenous theophylline and enprofylline against histamine- and adenosine 5'-monophosphate-provoked bronchoconstriction: implications for the mechanisms of action of xanthine derivatives in asthma. Pulm Pharmacol 1989; 2: 147-154.

92 Persson C, Uller L. HH Salter (1860s): taking cold as original cause and provocative of attacks of asthma. Thorax 2013; 68: 489.

93 Prinz F, Schlange T, Asadullah K. Believe it or not: how much can we rely on published data on potential drug targets? Nat Rev Drug Discov 2011; 10: 712. 